“C 2011 IEEE. Personal use of this material is permitted. Permission from IEEE must be obtained for all other uses, in any current or future media, including reprinting/republishing this material for advertising or promotional purposes, creating new collective works, for resale or redistribution to servers or lists, or reuse of any copyrighted component of this work in other works." 


\title{
Eddy-current Loss Prediction in the Rotor Magnets of a Permanent Magnet Synchronous Generator with Modular Winding Feeding a Rectifier Load
}

\author{
Jianning Dong ${ }^{1}$, Yunkai Huang ${ }^{1}$, Long $\mathrm{Jin}^{1}$, Jianguo Zhu², and Youguang Guo ${ }^{2}$ \\ ${ }^{1}$ School of Electrical Engineering, Southeast University, Nanjing, China \\ ${ }^{2}$ School of Electrical, Mechanical and Mechatronic Systems, University of Technology Sydney, Australia \\ Email: jnd.dong@gmail.com
}

\begin{abstract}
In a permanent magnet synchronous generator (PMSG) with modular winding, significant eddy-current may be induced in the rotor magnets due to asynchronous rotating stator magneto-motive forces (MMFs), and a rectifier load may signify the situation further. The eddy-current loss prediction in the rotor magnets of a permanent magnet synchronous generator with modular winding feeding a rectifier load is described. An analytical method considering the time harmonics of the stator currents and space harmonics of the stator MMFs and a time-stepping, coupled-circuit, two-dimensional (2D) finite-element method (FEM) are adopted. The predicted losses obtained from these two methods are compared and investigated.
\end{abstract}

Index Terms-Permanent magnet machines, Modular windings, Magnetic losses, Rectifier load

\section{INTRODUCTION}

$\mathrm{P}$ ERMANENT MAGNET synchronous generators (PMSGs) are increasingly adopted in distributed generation systems and electric vehicles for their high efficiency and high power density. In these applications, PMSG is often connected to a diode rectifier. The rectifier can then be connected to the power grid or hybrid electric vehicle motor through inverter. The rectifier load, in return, introduces significant current harmonics in the generator [1].

A new topology of PM brushless machine referred as "modular", whose slot number $N_{s}$ and pole number $2 p_{r}$ are related by $2 p_{r}=N_{s}-2$, emerged several years ago. When operating as a generator, the "modular" machine offers a number of attractive advantages such as higher power capacity and lower torque ripple over the traditional PM brushless machines [2]-[3]. When used in the generator to further increase the power capacity, the relatively high conductivity of sintered neodymium-iron-boron $(\mathrm{NdFeB})$ magnets may give rise to eddy current loss significant enough to result in partial irreversible demagnetization of the magnets [4]-[5]. This situation is aggravated further when the modular PMSG is connected to a rectifier load. The modular PMSG, which has nearly equal pole and slot number combinations, has a stator magneto-motive force (MMF) containing a large number of space harmonics [3], which cause eddy current loss in the magnets. The time harmonics of the phase current introduced by the rectifier load signify the eddy current loss even further.

Eddy-current loss in the rotor magnets can be predicted by employing time-stepped finite element analysis (FEA), both 2D and 3D [5]-[7]. However, these methods are time consuming and not intuitive enough for theoretical analysis. The analytical method presented in this paper focuses on magnet eddy current loss caused by stator MMF space harmonics and phase current time harmonics introduced by the rectifier load [8]-[9]. It is applied to a system composed of an out-rotor three-phase 18-slot/16-pole modular permanent magnet synchronous generator and a diode rectifier bridge with a capacitor filter. The analytically predicted eddy-current loss is validated by the result of a coupled field-circuit 2D time-stepped FEA. Winding distributions of the generator are showed in Fig. 1. As can be seen, the coils of each phase are wound on adjacent teeth, which is the key feature of the modular machine [2]. Parameters of the generator-rectifier system are showed in Table I.

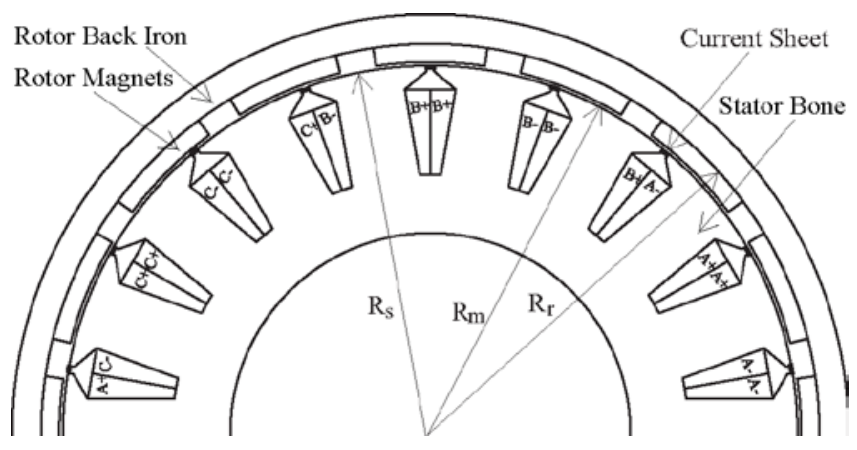

Fig. 1 Winding distributions for the 18-slot/16-pole modular PMSG (half) and the equivalent current sheet

TABLE I

PARAMETERS OF THE PMSG-RECTIFIER SYSTEM

\begin{tabular}{ll}
\multicolumn{2}{c}{ PARAMETERS OF THE PMSG-RECTIFIER SYSTEM } \\
\hline \hline Parameter & Value \\
\hline Pole number, $2 p_{r}$ & 16 \\
Slot number, $N_{s}$ & 18 \\
Stack length, $l_{a}$ & $50 \mathrm{~mm}$ \\
Slot opening, $b_{0}$ & $2 \mathrm{~mm}$ \\
Stator bone radius, $R_{s}$ & $101 \mathrm{~mm}$ \\
Magnet inner radius, $R_{m}$ & $102 \mathrm{~mm}$ \\
Magnet outer radius, $R_{r}$ & $107 \mathrm{~mm}$ \\
Magnet resistivity, $\rho$ & $1.5 \mathrm{e}-6 \Omega \mathrm{m}(\Omega / \mathrm{m} ?)$ \\
Series turns per phase, $N_{p h}$ & 48 \\
Pole arc/pitch ratio & 0.75 \\
Rectifier capacitor $C_{F}$ & $750 \mu \mathrm{F}$ \\
\hline \hline
\end{tabular}

\section{ANALYTICAL METHOD}

In this method, all the eddy current loss in the magnets is assumed to be caused by armature reaction field, i.e., the effect of stator slotting and the modifying effect of the induced eddy currents on the magnetic field are neglected. The stator 
and rotor iron cores are assumed to be infinitely permeable and the magnets have relative recoil permeability of $\mu_{m}=1$. Since the skin depth is greater than both the pole arc and the radial thickness of the magnets due to their relatively high electrical resistivity and low recoil permeability, the induced eddy currents in the magnets are assumed to be resistance limited. To obtain the armature reaction field distribution, the stator winding is modeled as equivalent current sheets distributed over the stator slot openings [10], as is showed in Fig. 1. Fig. 2 shows current density of each current sheet of phase A.

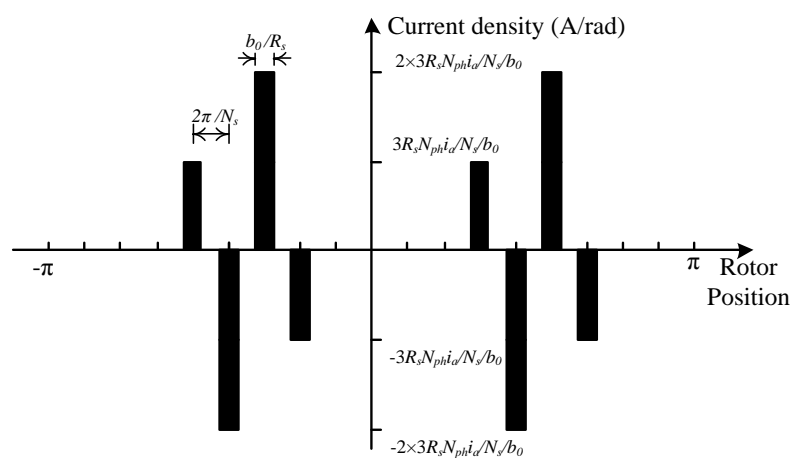

Fig. 2 Current sheet distribution of Phase A winding

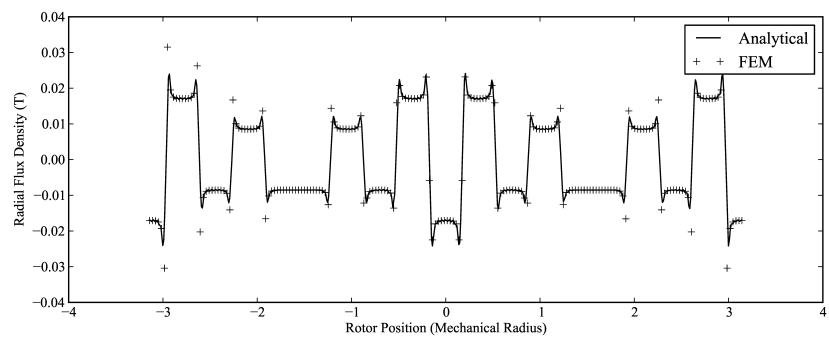

Fig. 3 Radial armature reaction air-gap flux density

The Fourier series expression for the equivalent current sheets of the three-phase winding in the rotating polar coordinate is

$$
\begin{aligned}
J_{a b c}\left(\theta, R_{s}, t\right) & =\sum_{u}^{\infty} \sum_{v}^{\infty} \mp \frac{2 N_{p h} I_{u}}{\pi R_{s}} K_{s o v} K_{w v} \times \\
& \cos \left[\left(u p_{r} \pm v p_{s}\right) \omega_{r} t \pm v p_{s} \theta_{r}+\theta_{u}\right]
\end{aligned}
$$

where $u$ is the time-harmonic order of the phase currents, $v$ is the space-harmonic order of the stator MMF. They are related by $v=3 c \pm u$, where $c$ is an integer. $I_{u}$ is the amplitude of the harmonic phase current, $p_{r}$ and $p_{s}$ are number of pole pairs of rotor and stator, respectively, viz. 8 and 1 on this occasion. $\omega_{r}$ is the rotor angular velocity, $\theta_{u}$ is the phase angle of the current harmonic, $K_{\text {sov }}$ is the slot opening factor [10], $K_{w v}$ is the winding factor. For an 18-slot/16-pole machine, $K_{w v}$ can be solved from Fig. 2, viz.:

$$
\begin{aligned}
K_{w v}= & \frac{1}{3}\left[0.5 \times \sin \left(\frac{3 \times 2 v \pi}{N_{s}}\right)-\sin \left(\frac{4 \times 2 v \pi}{N_{s}}\right)\right. \\
& \left.+\sin \left(\frac{5 \times 2 v \pi}{N_{s}}\right)-0.5 \times \sin \left(\frac{6 \times 2 v \pi}{N_{s}}\right)\right]
\end{aligned}
$$

The magnetic vector potential distribution in the magnet and air-gap $A\left(r, \theta_{r}, t\right)$ can be derived by solving the Laplace's equation (neglecting the modifying effect of the induced eddy currents on the magnetic field):

$$
\nabla^{2} A=0
$$

whose boundary conditions are

$$
\left.B_{\theta}\right|_{r=R_{r}}=0 \text { and }\left.H_{\theta}\right|_{r=R_{s}}=J_{a b c}
$$

where $B_{\theta}$ and $H_{\theta}$ are the circumferential components of the flux density and magnetic field strength, respectively. Fig. 3 compares the radial air-gap flux density solved from (1)-(4) with that obtained from FEM. Good agreements are achieved except some small deviations due to slot openings.

The induced eddy currents in the magnets can then be given by

$$
J_{m}\left(r, \theta_{r}, t\right)=-\frac{1}{\rho} \frac{\partial A}{\partial t}+C(t)
$$

where $C(t)$ is a constant independent of $r$ or $\theta_{r}$ which guarantees that the net total current flowing in each magnet segment is zero at any instant, i.e.,

$$
\int_{-\frac{\alpha_{p}}{2}}^{\frac{\alpha_{p}}{2}} \int_{R_{m}}^{R_{r}} J_{m} r \mathrm{~d} r \mathrm{~d} \theta_{r}=0
$$

where $R_{m}$ and $\alpha_{p}$ are the magnet inner radius and the angle of one magnet segment in radian respectively. Then the eddycurrent loss in all the magnet segments in the generator can be derived as

$$
P=2 p_{r} \frac{\omega_{r}}{2 \pi} \int_{0}^{l_{a}} \int_{0}^{\frac{2 \pi}{\omega_{r}}} \int_{\frac{-\alpha_{p}}{2}}^{\frac{\alpha_{p}}{2}} \int_{R_{m}}^{R_{r}} \rho J_{m}^{2} r \mathrm{~d} r \mathrm{~d} \theta_{r} \mathrm{~d} t \mathrm{~d} l
$$

where $l_{a}$ is the stack length of the generator. The final expression for numerical computation is given as:

$$
\begin{aligned}
P= & \frac{\mu_{0}^{2} \alpha_{p} p_{r} l_{a}}{\rho} \sum_{u}^{\infty} \sum_{v}^{\infty} \frac{\left(u p_{r} \pm v p_{s}\right)^{2} \omega_{r}^{2}}{v^{2} p_{s}^{2}} \\
& \times\left\{\left(\frac{R_{s}}{R_{m}}\right)^{2 v p_{s}} R_{s}^{2} R_{m}^{2} F_{v}+\left(\frac{R_{s}}{R_{r}}\right)^{2 v p_{s}} \frac{R_{s}^{2} R_{r}^{2}}{2 v p_{s}+2}\right. \\
& \times\left[1-\left(\frac{R_{m}}{R_{r}}\right)^{2 v v_{s}+2}\right]+\left(\frac{R_{s}}{R_{r}}\right)^{2 v p_{s}}\left(R_{r}^{2}-R_{m}^{2}\right) \times \\
& -\frac{8 \mu_{0}^{2} p_{r} l_{a}}{\alpha_{p} \rho} \sum_{u}^{\infty} \sum_{v}^{\infty} \frac{\left(u p_{r} \pm v p_{s}\right)^{2} \omega_{r}^{2}}{v^{4} p_{s}^{4}} \\
& \left.\times\left\{\left(\frac{R_{s}}{R_{r}}\right)^{2 v v_{s}}\right]^{-2}\right\} \times\left(\frac{3 N_{p h} I_{u} K_{s o v} K_{w v}}{\pi R_{s}}\right)^{2} E_{v}+\left(\frac{R_{s}}{R_{r}}\right)^{v p_{s}} \frac{R_{s} R_{r}^{2}}{2+v p_{s}} \\
& \left.\times\left[1-\left(\frac{R_{m}}{R_{r}}\right)^{v p_{s}+2}\right]\right\} \times \sin ^{2}\left(v p_{s} \frac{\alpha_{p}}{2}\right) / \\
& \left\{\left(R_{r}^{2}-R_{m}^{2}\right)\left[1-\left(R_{s} / R_{r}\right)^{2 v p_{s}}\right]^{2}\right\} \\
& \times\left(3 N_{p h} I_{u} K_{s o v} K_{w v} / \pi / R_{s}\right)^{2}
\end{aligned}
$$

where 


$$
F_{v}= \begin{cases}\frac{\left(R_{r} / R_{m}\right)^{2-2 v p_{s}}-1}{2-2 v p_{s}}, & \text { if } v p_{s} \neq 1 \\ \ln \left(R_{r} / R_{m}\right), & \text { if } v p_{s}=1\end{cases}
$$

and

$$
E_{v}= \begin{cases}\frac{\left(R_{r} / R_{m}\right)^{2-v p_{s}}-1}{2-v p_{s}}, & \text { if } v p_{s} \neq 2 \\ \ln \left(R_{r} / R_{m}\right), & \text { if } v p_{s}=2\end{cases}
$$

\section{COUPLED FIELD-CIRCUIT TIME-STEPPED FEM}

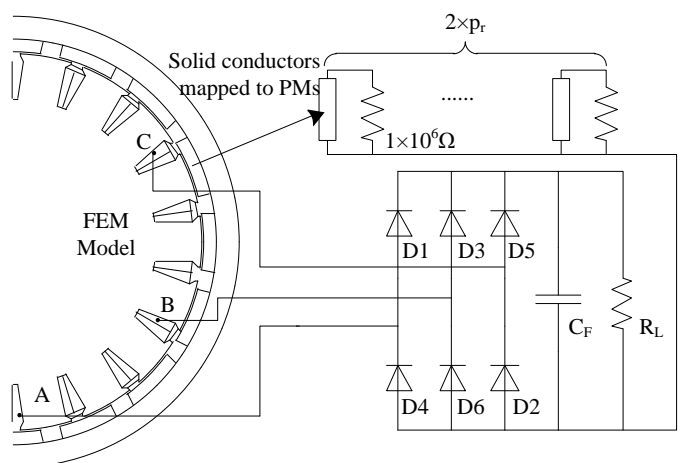

Fig. 4 Coupled field-circuit FEM model of the generator-rectifier system

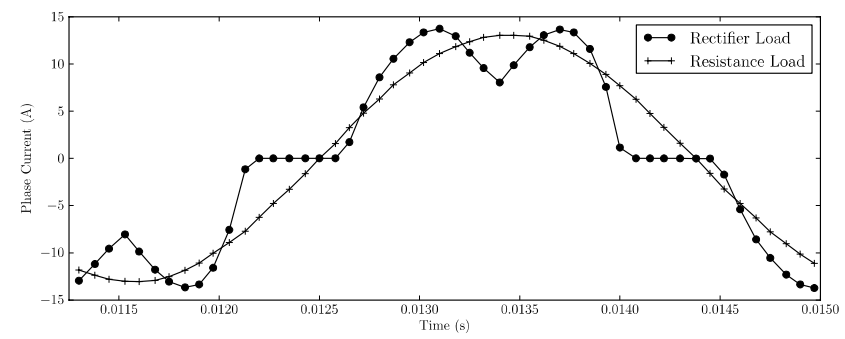

(a) Simulated current waveform of phase A

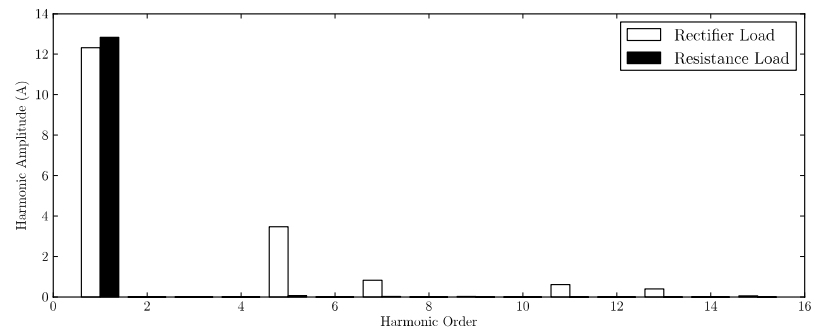

(b) Spectrum of simulated phase A current waveform

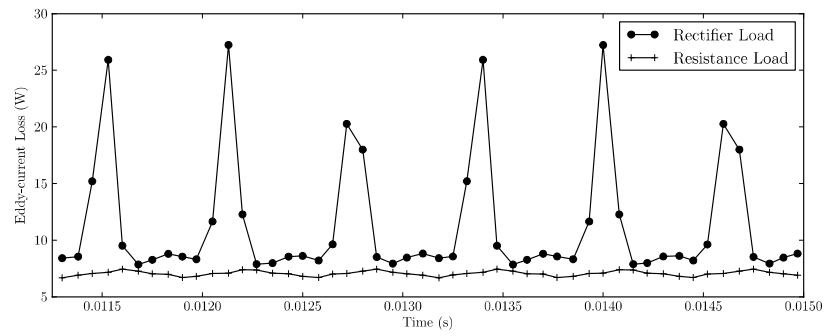

(c) Simulated instantaneous eddy-current loss in all the magnets Fig. 5 Phase current and magnets loss calculated by FEM at 2000 rpm, 1900 $\mathrm{W}$

The coupled field-circuit FEM model used for computation of eddy-current loss in the magnets is showed in Fig. 4. In this model, permanent magnets are defined as linear magnets described by the $B_{r}$ module with isotropic resistivity. These magnets are coupled with solid conductors in the external circuit. Large resistor (1e6 $\Omega$ ) is connected in parallel with each solid conductor since the "terminals" of each magnet segment are open (see Fig. 4) [11]. 60085 nodes and 29962 elements are used in the time-stepped 2-D transient solver. Each time-step is chosen as 1/50 of one electrical period, viz. 0.9 mechanical degrees. Steady-state phase current and corresponding spectrum (Fig. 5a-5b), current density distribution in each magnet, and hence the eddy-current loss in all the magnets (see Fig. 5c, obtained from (7)) are obtained from the FEM results. Operation of the generator when feeding pure resistance load is simulated in the same way for further investigation.

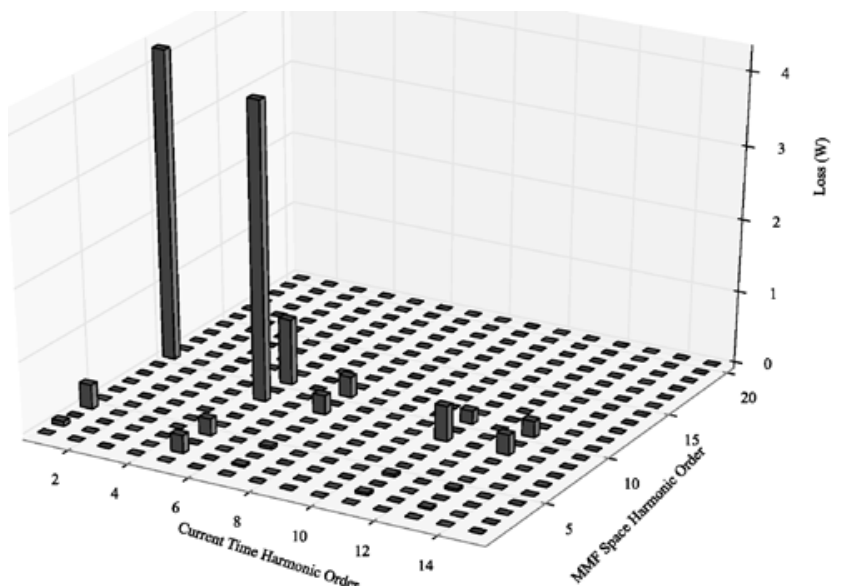

Fig. 6 Loss spectrum calculated by the analytical method at 2000 rpm, 1900 $\mathrm{W}$, rectifier load

\section{COMPARISON AND INVESTIGATION}

The analytical method presented in (1)-(10) is carried out using a script written in Python [12]. By substituting the amplitudes of phase current time harmonics into the script, the eddy-current loss caused by each time-space harmonic pair is calculated and plotted, as is showed in Fig. 6. Then the total loss can be obtained by summarizing all the loss harmonics.

TABLE II

Losses Calculated by FEM and Analytical Method at Various Operating Points

\begin{tabular}{llllll}
\hline \hline \multirow{2}{*}{$\begin{array}{c}\text { Speed } \\
\text { (rpm) }\end{array}$} & \multirow{2}{*}{$\begin{array}{c}\text { Output } \\
\text { Power (W) }\end{array}$} & \multicolumn{3}{c}{ Loss in All Magnets (W) } \\
\cline { 3 - 6 } & & Rectifier Load & \multicolumn{2}{c}{ Resistance Load } \\
\cline { 3 - 6 } & & FEM & Analytical & FEM & Analytical \\
\hline \multirow{2}{*}{3000} & 1400 & 10.4 & 9.90 & 2.98 & 2.64 \\
\cline { 2 - 6 } & 2960 & 23.4 & 28.0 & 15.0 & 14.0 \\
\hline \multirow{2}{*}{2000} & 960 & 5.50 & 4.90 & 1.54 & 1.24 \\
\cline { 2 - 6 } & 1900 & 11.3 & 12.2 & 7.05 & 6.07 \\
\hline \multirow{2}{*}{1000} & 490 & 1.51 & 1.17 & 0.41 & 0.32 \\
\cline { 2 - 6 } & 960 & 3.40 & 3.30 & 1.84 & 1.49 \\
\hline \multirow{2}{*}{500} & 185 & 0.31 & 0.20 & 0.06 & 0.05 \\
\cline { 2 - 6 } & 350 & 0.57 & 0.47 & 0.24 & 0.18 \\
\hline \hline
\end{tabular}

Table II compares eddy-current losses in the magnets calculated by FEM and the presented analytical method when the generator feeds a rectifier load or a pure resistance load. It can be seen from the table that the analytically calculated eddy-current loss is smaller than the loss obtained by FEM under most cases. The deviation is caused by air-gap permeance variation due to stator slotting and iron saturation, and additional time and space harmonics taken into 
consideration in FEM. However, when the generator is operating at high speed feeding a rectifier load, the analytically calculated result is smaller compared with that acquired from FEM. The induced eddy currents in the magnets are not resistance limited due to the skin effect of the magnets when the generator operating at high speed:

The skin depth of the magnets can be calculated from:

$$
\delta=\sqrt{\frac{2 \rho}{\left(u p_{r} \pm v p_{s}\right) \omega_{r} \mu_{0} \mu_{r}}}
$$

It can be seen from Fig. 6 that the time-space harmonic pair of $u=1, v=10$ contributes the most to the eddy-current loss among all the pairs when the generator feeds a rectifier load. For this harmonic pair, the skin depth of the magnets can be got from (11), viz. $\delta=25 \mathrm{~mm}$ when $n=2000 \mathrm{rpm}$. This is smaller than the pole arc, which is about $30 \mathrm{~mm}$. Skin depth of the fifth time harmonic and corresponding effective space harmonics $(v=2,4,6,8 \ldots)$, which contribute the largest part to the loss, is even smaller. Hence the assumption that all the eddy currents are resistance limited, made when deriving the analytical model, is not valid any more.

The net eddy-current loss in the magnets produced by the eighth space harmonic with the fundamental time harmonic is zero since it rotates synchronously with the rotor to produce the electromagnetic torque $\left(u p_{r} \pm v p_{s}=0\right)$, as seen in Fig. 6 .

The rectifier load introduces significant time harmonics, especially the fifth harmonic, into the phase current, as is showed in Fig. 5a. These phase current time harmonics, in return, induce significant eddy-current loss in the magnets, which can be seen from Fig. 5c, Fig. 6 and Table II. By comparing Fig. 5a with Fig. 5c, it can be seen that the instantaneous eddy-current loss in the magnets increases sharply near the commutation moment of the rectifier. Hence the loss can be reduced by adopting better rectifying schemes which can suppress the phase current time harmonics such as PWM rectifying [13].

Another method to reduce the magnet loss is magnet segmenting [8]. This method can be derived from (8): since $P$ is proportional to $\alpha_{p}$, the loss is inversely proportional to number of segments per pole arc. Table III shows the effect of circumferentially segmenting of magnets on eddy-current loss predicted by FEM. It can be seen that this method is very effective.

TABLE III

MAGNET EDDY-CURRENT LOSS REDUCTION BY CIRCUMFERENTIALLY SEGMENTING, 1000 RPM, $960 \mathrm{~W}$, RECTIFIER LOAD

\begin{tabular}{lllll}
\hline \hline Segments/pole & 1 & 2 & 3 & 4 \\
\hline Eddy-current loss & 3.40 & 0.61 & 0.22 & 0.13 \\
\hline \hline
\end{tabular}

\section{CONCLUSION}

This paper presents an analytical method to predict the eddy-current loss in the rotor magnets of a PMSG feeding a rectifier load. The analytically calculated eddy-current loss and that obtained from time-stepping, coupled-circuit, 2D FEM are compared and investigated.

The causes of deviations between these two methods are analyzed. Two effective ways to reduce the magnet loss are raised based on the analysis of the results. The next steps are to develop a time-efficient model of eddy-current loss in the magnets and efficiency of PMSG when feeding a PWM rectifier load. Control scheme of the PWM rectifier to maximize the efficiency of the PMSG while reducing the eddy-current loss in the magnets to reasonable level will be developed based on this model.

\section{REFERENCE}

[1] T.-F. Chan, W. Wang, and L. L. Lai, "Coupled field-circuit analysis of a surface-inset permanent-magnet synchronous generator feeding a rectifier load," in Proc. IEEE Int. Electric Machines and Drives Conf. IEMDC '09, 2009, pp. 1600-1604.

[2] J. Wang, K. Atallah, Z. Q. Zhu, and D. Howe, "Modular three-phase permanent-magnet brushless machines for in-wheel applications," IEEE Trans. Veh. Technol., vol. 57, no. 5, pp. 2714-2720, Sep. 2008.

[3] J. Wang, Z. P. Xia, D. Howe, and S. A. Long, "Comparative study of 3phase permanent magnet brushless machines with concentrated, distributed and modular windings," in Proc. 3rd IET Int Power Electronics, Machines and Drives Conf, 2006, pp. 489-493.

[4] K.-C. Kim, K. Kim, H. J. Kim, and J. Lee, "Demagnetization analysis of permanent magnets according to rotor types of interior permanent magnet synchronous motor," IEEE Trans. Magn., vol. 45, no. 6, pp. 2799-2802, 2009.

[5] K. Yamazaki, M. Shina, Y. Kanou, M. Miwa, and J. Hagiwara, "Effect of eddy current loss reduction by segmentation of magnets in synchronous motors: Difference between interior and surface types," IEEE Trans. Magn., vol. 45, no. 10, pp. 4756-4759, 2009.

[6] T. Okitsu, D. Matsuhashi, and K. Muramatsu, "Method for evaluating the eddy current loss of a permanent magnet in a PM motor driven by an inverter power supply using coupled 2-D and 3-D finite element analyses," IEEE Trans. Magn., vol. 45, no. 10, pp. 4574-4577, 2009.

[7] W. N. Fu and Z. J. Liu, "Estimation of eddy-current loss in permanent magnets of electric motors using network-field coupled multislice timestepping finite-element method," IEEE Trans. Magn., vol. 38, no. 2, pp. 1225-1228, 2002.

[8] K. Atallah, D. Howe, P. H. Mellor, and D. A. Stone, "Rotor loss in permanent-magnet brushless AC machines," IEEE Trans. Ind. Appl., vol. 36, no. 6, pp. 1612-1618, 2000.

[9] Z. Q. Zhu, K. Ng, N. Schofield, and D. Howe, "Improved analytical modeling of rotor eddy current loss in brushless machines equipped with surface-mounted permanent magnets," IEE Proceedings -Electric Power Applications, vol. 151, no. 6, pp. 641-650, 2004.

[10] Z. Q. Zhu and D. Howe, "Instantaneous magnetic field distribution in brushless permanent magnet dc motors. ii. armature-reaction field," IEEE Trans. Magn., vol. 29, no. 1, pp. 136-142, 1993.

[11] Cedrat, "Software solutions: Flux," CEDRAT Group,OCT 2010,. [Online]. Available: http://www.cedrat.com/en/softwaresolutions/flux.html

[12] E. Jones, T. Oliphant, P. Peterson et al., "SciPy: Open source scientific tools for Python,” 2001-2010. [Online]. Available: http://www.scipy.org/

[13] H. Bai, F. Wang, and J. Xing, "Control strategy of combined PWM rectifier/inverter for a high speed generator power system," in Proc. $2^{\text {nd }}$ IEEE Conf. Industrial Electronics and Applications ICIEA 2007, 2007, pp. 132-135. 